certingly the other four all failed to single out enough of these 'false' values. There was some agreement among those present at the Washington meeting that dioxin values close to the detection limit - regarded as being 9 ppt-did complicate matters and that perhaps some false positives and negatives were unavoidable.

In future, the researchers will probably abide by the gentleman's agreement practised so far that only values which are at least $2 \frac{1}{2}$ times greater than the noise levels will be regarded as true measurements. This decision could well eliminate some of the false predictions. Some scientists-notably those at Dow -feel that dioxin estimations lower than $9 \mathrm{ppt}$ must, therefore, still be treated with scepticism. Others still disagree, and argue that it depends on the methods of estimation.

Contamination of the dioxin extracts by other chlorinated hydrocarbonsPCBs, for example-makes interpre- tation more difficult. Dow argue that this could be one reason for their poor results and that their extraction would produce a "cleaner" sample. However, contamination is not the only problem. There is still no measure of agreement between the practitioners on the methods to be used to estimate the recovery of the final dioxin sample.

Dioxin chlorinated with ${ }^{37} \mathrm{Cl}$ is used in most cases for this purpose: the tracer is normally incubated with samples before extraction, but there are still doubts in the minds of some of the researchers that this tracer 'equilibrates' with the natural dioxin to a sufficient extent so that estimates of the final recovery are indeed accurate.

There are doubts too about mass spectrometry readings and views differ as to whether two dioxin ions with a selected mass from natural dioxin $(320 \mathrm{~m} / \mathrm{e}, 322 \mathrm{~m} / \mathrm{e})$ should be measured and the results averaged or whether one mass reading is sufficient. The results in the EPA review are more or less independent of the method of measurement-good and poor results were recorded by both mass spectrometry methods.

Although some of these views have still to be resolved there was still general agreement at the meeting that the EPA should publish the results of this collaborative programme as soon as possible and that it should be subject to peer review. However, whether the reviewers agree with the consensus of opinion expressed at the meeting that dioxin analysis has become far better in the past few years remains to be seen.

What they will undoubtedly say is that there ought to be even more collaboration between laboratories to ensure greater reproducibility of results. As for the EPA, it will probably be given credit for initiating the first major stage in this collaboration.

Alastair Hay

\title{
Chemicals in food: new US framework proposed
}

A NEW framework for regulating toxic substances in food, which would give greater discriminatory power to the Food and Drug Administration, and allow the benefits as well as the risks of a particular substance to be taken into account, has been suggested by a study group of the National Academy of Sciences.

The proposal is made in a report published last Friday by the Academy's Institute of Medicine and National Research Council, prepared at the request of Congress following the controversy two years ago over whether or not saccharin is carcinogenic, and if so what should be done about it.

According to the panel of scientists, lawyers and public policy experts which prepared the report, the proposed framework-under which a substance could be placed in a category of high, moderate or low risk, with discrimination given to the FDA over handling substances in each category-would effectively separate the scientific assessment of risk from its social consequence.

In a letter submitting the report to Health Secretary Mr Joseph Califano, for example, Dr Philip Handler, president of the academy, says: "estimation of risk is a scientific matter, albeit not always readily feasible. Decision concerning the acceptability and management of a given risk is an intrinsically political question to be returned to the polity for determination."

Not all of the panel, however, agreed that the distinction is a tenable one. A minority report, disputing the suggestion that toxic food substances can be divided into three categories according to varying degrees of risk, says that there is "no scientifically defensible way" of doing this.

The statement, signed by five of the 37 members of the committees responsible for the report, says that "the ability of science to quantify human risk has not advanced sufficiently since the formulation of the Delaney Amendment [which banned all food additives shown to cause cancer in laboratory animals] to permit the construction of a scientific rationale for such a scheme."

The report forms half of a two-part study carried out by the NAS under the terms of an act passed by Congress in 1977, which placed an 18-month moratorium on a ban on saccharin following the disclosure that it had been found carcinogenic in laboratory animals. The first part of the report was published last November, and concluded that saccharin was indeed a potential human carcinogen, although its potency was probably low compared to other known cancer-causing agents, such as cigarettes.

The second report is concerned with the public policy implications of this and similar findings for the regulation of food safety. It recommends a single policy applicable to all foodstuffs, additives and contaminants, and says that regulatory agencies should be able to do more than simply ban or not ban a particular substance.

"This report suggests that a realistic policy would be to weigh the estimated level of risk of a substance in our food supply against the perceived benefits of its use, and to employ informed judgment as a basis for regulatory decisions," according to Dr David A. Hamburg, president of the Institute of Medicine.

A major problem presented by current law, the report says, is that most cases of both health and nonhealth benefits are excluded from regulatory consideration. "It is better to have benefits defined, evaluated and openly considered when that is possible", it says.

Speaking in Washington last week Professor Don K. Price, one of the panel members responsible for the report, denied that by shifting the focus of decision-making from Congress to a regulatory agency, the process would become more vulnerable to outside pressures. "The committee felt that the present scheme, which in theory asks the FDA to make a purely scientific decision, has not worked very well, and in many cases political and other considerations have been smuggled in-or, as in the saccharin case, have given rise to other problems", he said.

"This new system would require regulatory agencies to get a statement on the scientific aspects from a research institution, and the subsequent decision-making process can then be made clear, public and open, so that you can deal with it better than if you ignored outside pressure".

The committee made no particular recommendation for the marketing of saccharin, and the minority statement disagreed with the view of the majority that "a total immediate ban of saccharin would not be a sound regulatory step at the present time."

David Dickson 